\title{
Kinetic Characterization of Precipitation Reactions: Possible Link between a Phenomenological Equation and Reaction Pathway
}

\author{
Nirmali Prabha Das, Réka Zahorán, László Janovák, Ágota Deák, Ágota Tóth, Dezső Horváth, \\ and Gábor Schuszter*
}

Cite This: Cryst. Growth Des. 2020, 20, 7392-7398

Read Online

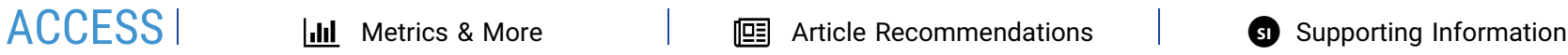

ABSTRACT: The characteristic time scale of reactive crystallization is traditionally investigated as a function of supersaturation in the deterministic regime, but no chemical meaning is assigned to the empirical power law. Applying chemical model systems in which various oxalate complexes form beside the precipitate, we show that the exponent provides information about the reaction pathways. The speciation of the reactant solution is revealed by combining equilibrium calculations and conductance measurements; the precipitate is identified with powder X-ray diffraction. A link between microstructure and kinetics is illustrated by scanning electron microscopy. The functionality of complex-shaped particles is examined by utilizing them as filler material to modify the wetting properties of a fluoropolymer-based thin film. Finally, it is shown that investigating the characteristic time scale as a function of the analytical concentration instead of supersaturation may also provide valuable information.

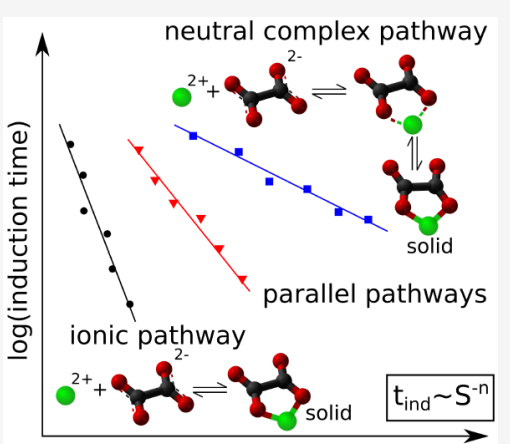

log(supersaturation)

\section{INTRODUCTION}

Improving the products of precipitation, i.e., reactive crystallization, is of interest from scientific and industrial perspectives as well. Prominent examples are antigen-antibody reactions, ${ }^{1}$ synthetic graft design, ${ }^{2,3}$ synthesis of superparamagnetic materials, ${ }^{4}$ layered double hydroxides, ${ }^{5}$ pharmaceuticals, pigments, metal oxides, etc. ${ }^{6}$ Although the reactions are traditionally carried out in one-pot systems, dynamic processes extensively building on the coupling of transport phenomena and reactions may provide an enhanced control of the product properties. Size-controlled metal-organic frameworks, ${ }^{7}$ polymorph selection in flow, ${ }^{8,9}$ bioenergetics of membranes, ${ }^{10}$ and underground carbon dioxide sequestration $^{11,12}$ are also studied this way.

Time scale matching of the various transport processes and chemical reactions is required to perform a tailored synthesis. The thermodynamics of precipitation has been thoroughly studied; various theories have been elaborated to explain nucleation in either classical or nonclassical ways. ${ }^{13,14}$ However, less is known about the relationship of kinetic descriptors and reaction mechanism. ${ }^{6}$ The characteristic time scale of a precipitation reaction is often described by the induction period $\left(t_{\text {ind }}\right)$, which measures the time elapsed between maintaining supersaturation $(S)$ and detecting precipitation. ${ }^{15}$ Although that parameter and its stochastic or deterministic feature depends on the apparent supersaturation, ${ }^{16}$ system size, and detection technique, it can be reproducibly determined by various methods. ${ }^{17-19}$ Throughout our work, the determinstic induction period is measured, i.e. such experimental conditions are maintained, where $t_{\text {ind }}$ involving multiple nucleation and growth is reproducible rather than is given by a distribution. Numerous theoretical formulas have been derived on the basis of thermodynamic parameters to express $t_{\text {ind }}$ as a function of supersaturation. ${ }^{20,21}$ Such equations can be used to approximate the proportion of nucleation time and the time required for a critical nucleus to grow and reach a detectable size by the apparent technique. Information about the magnitude of thermodynamic parameters (e.g., surface energy) and the way of crystal growth (e.g., mononuclear or polynuclear) also can be obtained. However, no reference has been provided about the mechanism of the chemical reaction, especially when parallel pathways are available.

The kinetics of a precipitation reaction is often characterized by the phenomenological equation

$$
t_{\text {ind }}=a_{S} S^{-n_{S}}
$$

however, no fundamental significance is assigned to $n_{S}$, as declared in specialized textbooks: "The exponent $n_{S}$, which is frequently referred to as the apparent order of nucleation, has no fundamental significance. It does not give an indication of the number of elementary species involved in the nucleation process." 22 A similar conclusion has been drawn in relevant scientific

Received: July 30, 2020

Revised: October 12, 2020

Published: October 22, 2020 
papers as well. ${ }^{20,21}$ At the same time, it is observed that $n_{S}$ varies from one reaction to another. ${ }^{6}$ The product of precipitation is usually a sparingly soluble salt; thus, $S$ reaches a high value in the reaction mixture. Nucleation plays an important role and leads to the formation of a vast amount of tiny crystals. Both homo- and heterogeneous nucleation processes are relevant, but secondary nucleation can usually be neglected. ${ }^{6}$

In this context, the aim of our study is to understand what lies behind the different time scale of very similar precipitation reactions and thus to find the chemical meaning of the empirical rate law. The results are organized as follows. First, we identify the reaction products in order to facilitate the determination of solution speciation by combining equilibrium calculations and conductance measurements. The apparent speciation accounts for supersaturation, which is then used for kinetic fits. Furthermore, the power laws are rendered to possible reaction pathways and the link between exponents and particle surface features is presented. Finally, a potential application of the complex microstructures is shown.

\section{RESULTS AND DISCUSSION}

Precipitation kinetics experiments are performed where $t_{\text {ind }}$ is determined through time-elapsed turbidity $(T)$ measurements for various chemical systems and reactant concentrations in a well-stirred spectrophotometric cuvette (see the Supporting Information). The investigated systems are divided into two groups. An aqueous solution of either an alkaline-earth-metal $(\mathrm{Mg}, \mathrm{Ca}, \mathrm{Sr}$, and $\mathrm{Ba})$ or a transition-metal $(\mathrm{Co}, \mathrm{Ni}, \mathrm{Cu}, \mathrm{Zn}$, and $\mathrm{Cd}$ ) salt is mixed with a solution of a common precipitant $\left(\mathrm{Na}_{2} \mathrm{C}_{2} \mathrm{O}_{4}\right)$ in order to yield a precipitate. The end of the induction period is tracked by the sudden increase of the $T-t$ curve. To quantitatively determine $t_{\text {ind }}$, the baseline of the $T-t$ data set, where no turbidity increase can be detected, is linearly fitted and the error of the measurement (i.e., the standard deviation of the recorded points) is calculated. In the next step, a high-order polynomial is fitted to the ascending part of the data set. The end of the induction period is the instance when the polynomial overcomes the linear by 3 times the measurement error, as in standard analytical chemistry methods. ${ }^{18}$ The similarities and differences of the reactions of chemically similar metal ions and of the apparent kinetic orders are investigated to reveal information about the chemical meaning of $n_{S}$.

As a first step, since various oxalate complexes are expected to be present in the reaction mixture, ${ }^{23}$ those chemical species are sought to which the supersaturation can be attributed and thus which can act as precursors for precipitation. In this context, powder X-ray diffraction (XRD) measurements are carried out to identify the products. Experimental details and the obtained diffractograms are presented in the Supporting Information. The crystalline phases are determined with QUALX2 software $^{24}$ for most systems ( $\mathrm{Mg}, \mathrm{Ca}, \mathrm{Sr}, \mathrm{Ba}, \mathrm{Co}$, $\mathrm{Ni}, \mathrm{Cu}$, and $\mathrm{Cd}$ ), while $\mathrm{Zn}$ is classified with the additional use of published data. ${ }^{25}$ Importantly, according to the assigned diffractograms, each reaction provided a $\mathrm{MC}_{2} \mathrm{O}_{4}\left(\mathrm{H}_{2} \mathrm{O}\right)_{x}$ precipitate where $\mathrm{M}$ stands for the corresponding alkalineearth- or transition-metal ion and $x$ denotes the number of incorporated water molecules. $x$ varies from 0 to 3.5 for the different compounds. A single phase is found in each chemical system with the exception of $\mathrm{Sr}$ and $\mathrm{Ba}$ cases, where a mixture of two and five phases with different amounts of constitutional water are simultaneously present, respectively.
To further identify the precipitate precursor(s) in the different chemical systems, equilibrium calculations provide the probable speciation of the reaction mixture before the onset of precipitation, assuming a fast complex formation. The calculations are performed with Wolfram Mathematica by numerically solving the corresponding algebraic equation system.

The first and second protonation steps of the oxalate ions gained from the dissolution of $\mathrm{Na}_{2} \mathrm{C}_{2} \mathrm{O}_{4}$ salt are described as

$$
\begin{aligned}
& \mathrm{C}_{2} \mathrm{O}_{4}^{2-}+\mathrm{H}^{+} \rightleftharpoons \text { HOOC }-\mathrm{COO}^{-} \\
& \mathrm{HOOC}-\mathrm{COO}^{-}+\mathrm{H}^{+} \rightleftharpoons \mathrm{C}_{2} \mathrm{H}_{2} \mathrm{O}_{4}
\end{aligned}
$$

The metal ions $\left(\mathrm{M}^{2+}\right)$ may form water-soluble complexes both with the different oxalate species and with the hydroxide ions according to the equations

$$
\begin{aligned}
& \mathrm{M}^{2+}+\mathrm{C}_{2} \mathrm{O}_{4}^{2-} \stackrel{\beta_{1}}{\rightleftharpoons} \mathrm{MC}_{2} \mathrm{O}_{4}(\mathrm{aq}) \\
& \mathrm{M}^{2+}+2 \mathrm{C}_{2} \mathrm{O}_{4}^{2-} \stackrel{\beta_{2}}{\rightleftharpoons}\left[\mathrm{M}\left(\mathrm{C}_{2} \mathrm{O}_{4}\right)_{2}\right]^{2-} \\
& \mathrm{M}^{2+}+\mathrm{HOOC}-\mathrm{COO}^{-} \rightleftharpoons[\mathrm{M}(\mathrm{HOOC}-\mathrm{COO})]^{+} \\
& \mathrm{M}^{2+}+2 \mathrm{HOOC}-\mathrm{COO}^{-} \rightleftharpoons \mathrm{M}(\mathrm{HOOC}-\mathrm{COO})_{2}(\mathrm{aq}) \\
& \mathrm{M}^{2+}+x \mathrm{OH}^{-} \rightleftharpoons\left[\mathrm{M}(\mathrm{OH})_{x}\right]^{2-x}
\end{aligned}
$$

where $x$ varies between 1 and 4 for the abundant hydroxide complexes under mild conditions. Although a few other hydroxide complexes are also known in the literature, they are not relevant because of the $\mathrm{pH}$ provided during the reactions. Finally, the formation of the oxalate precipitates is characterized by the solubility product $\left(K_{\mathrm{sp}}\right)$ of the reaction

$$
\mathrm{M}^{2+}+\mathrm{C}_{2} \mathrm{O}_{4}^{2-} \stackrel{K_{\mathrm{sp}}^{-1}}{\rightleftharpoons} \mathrm{MC}_{2} \mathrm{O}_{4}(\mathrm{~s})
$$

A comprehensive list of the chemical equilibria taken into account together with the corresponding equilibrium constants $\left(\beta_{1}, \beta_{2}, K_{\mathrm{sp}}\right.$, etc. $)$ and the calculated $\mathrm{pH}$-dependent speciation for various chemical systems and concentrations are tabulated in the Supporting Information. As a result of the calculations, it is found that calcium and strontium are almost exclusively present in the free $\mathrm{M}^{2+}$ ion form. The $\mathrm{M}^{2+}$ ion is dominant for barium and cadmium as well, but some $\mathrm{MC}_{2} \mathrm{O}_{4}(\mathrm{aq})$ monooxalate complex is also obtained. The concentration of $\mathrm{MC}_{2} \mathrm{O}_{4}(\mathrm{aq})$ species increases in the cobalt, copper, nickel, magnesium, and zinc systems, respectively. Finally, the $\left[\mathrm{M}\left(\mathrm{C}_{2} \mathrm{O}_{4}\right)_{2}\right]^{2-}$ dioxalate complex also forms in the last mentioned systems, most prominently in the case of nickel and copper.

Although equilibrium calculations may provide information about the apparent chemical speciation of the reaction mixture, the time scale required for the different systems to reach a complex formation equilibrium might be significantly different and might not be necessarily achieved before the onset of precipitation. Therefore, conductance $(G)$ measurements are performed to further investigate the speciation. Examples are shown in Figure 1 for two limiting cases: i.e., for the calciumoxalate system with high $\mathrm{M}^{2+}$ ion concentration and for the copper-oxalate system characterized by a significant amount of mono- and dioxalate complexes. Before the reactants are mixed, the conductivity of each stock solution is determined as a reference. $G$ is also obtained for the diluted reactant 


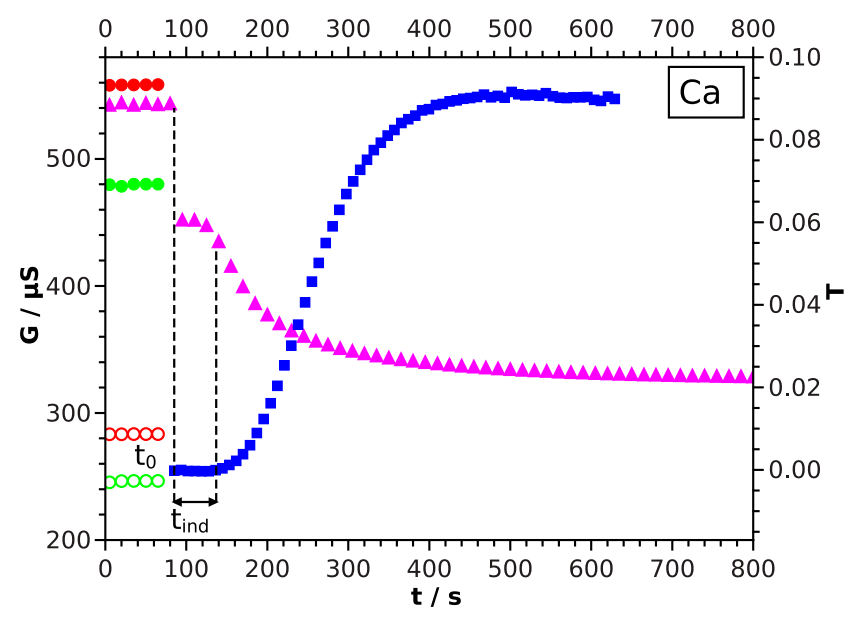

water-diluted solutions $(\sim 2400 \mu \mathrm{S})$, proving that most of the free ions, which contribute as precipitate compartments according to the XRD measurements, are already incorporated into various complexes. This agrees well with the equilibrium calculations and with the assumption of fast complex formation equilibria (see the Supporting Information for solution speciation). The absence of precipitate at the time when $G$ drops is further confirmed by the flat $T-t$ curve until the end of $t_{\text {ind. }}$. It is also found that $G$ stays technically constant $(2563$ $\pm 6 \mu \mathrm{S})$ over a long period of time $(\sim 1700 \mathrm{~s})$ even if precipitation takes place and provides a significant amount of product $(T \approx 2)$. Experimental details and further measurements are presented in the Supporting Information.

After performing the same analysis for each chemical system, i.e., compiling the results of the equilibrium calculations and conductance measurements, we find that the $\mathrm{M}^{2+}$ ion and the $\mathrm{MC}_{2} \mathrm{O}_{4}(\mathrm{aq})$ monooxalate complex can be considered as precipitate precursors in the case of calcium, strontium, barium, and cadmium, whereas the $\mathrm{MC}_{2} \mathrm{O}_{4}(\mathrm{aq})$ monooxalate and $\left[\mathrm{M}\left(\mathrm{C}_{2} \mathrm{O}_{4}\right)_{2}\right]^{2-}$ dioxalate complexes are relevant in the magnesium, cobalt, nickel, copper, and zinc cases (see the Supporting Information for the solution speciations). Therefore, the appropriate supersaturation $(S)$ can be defined for each reaction to fit according to eq 1 and to obtain the exponent $n_{S}$ for each precipitation system. Hereinafter, the actual concentrations are denoted with brackets, while equilibrium concentrations are marked with an additional superscript asterisk. According to the previously assigned precipitate precursors, $S=S_{\text {ion }}+S_{1^{\prime} 1}$ for the $\mathrm{Ca}, \mathrm{Sr}, \mathrm{Ba}$, and $\mathrm{Cd}$ systems, where $S_{\text {ion }}=\frac{\left[\mathrm{M}^{2+}\right]\left[\mathrm{C}_{2} \mathrm{O}_{4}^{2-}\right]}{K_{\mathrm{sp}}}$ describes the supersaturation according to the free ions and $S_{1^{\prime} 1}=\frac{\left[\mathrm{MC}_{2} \mathrm{O}_{4}(\mathrm{aq})\right]}{\beta_{1} K_{\mathrm{sp}}}$ refers to the supersaturation of the monooxalate complex. $S=S_{1^{\prime} 11}+S_{1^{\prime} 2}$ for the $\mathrm{Mg}, \mathrm{Co}, \mathrm{Ni}, \mathrm{Cu}$, and $\mathrm{Zn}$ systems, where $S_{1^{\prime} 2}=\frac{\left.\left[\mathrm{M}^{\prime}\left(\mathrm{C}_{2} \mathrm{O}_{4}\right)_{2}\right]^{2-}\right]\left[\mathrm{M}^{2+}\right]}{\mathrm{K}_{\mathrm{sp}}{ }^{\prime}}$ stands for the supersaturation of the dioxalate complex with $K_{\mathrm{sp}}{ }^{\prime}=\left[\left[\mathrm{M}\left(\mathrm{C}_{2} \mathrm{O}_{4}\right)_{2}\right]^{2-}\right] *\left[\mathrm{M}^{2+}\right]^{*}=$ $\beta_{2} K_{\mathrm{sp}}^{2}$. The deduction of the aforementioned expressions is given in the Supporting Information.

The measured induction periods are plotted as a function of solutions, in which the initial concentration is reduced to half by adding ion-exchanged water. Those $G$ values are used to designate the range of conductivity change during precipitation. In the $\mathrm{M}^{2+}$ ion dominated case (Figure 1-Ca), the initial conductance of the $\mathrm{CaCl}_{2}$ solution is measured first as a background. The oxalate solution is then added to and mixed with the $\mathrm{CaCl}_{2}$ solution at $t_{0}$, which is observed as a slight drop of $G$. For a short period of time $\left(t_{\text {ind }}\right), G$ only depicts a negligible decrease. Once the induction period is over, $G$ steeply decreases until the end of precipitation, where it reaches a constant value. The turbidity $(T)$ measured in separate experiments but with identical conditions is also illustrated in Figure 1-Ca to show that the onset of precipitation marked by the sudden increase in $T$ coincides with the conductivity measurement. The modest drop of $G$ when the reactants are mixed and its monotonous drop during precipitation highlight that ions are consumed during precipitation, as could be forecast with the aid of the equilibrium calculations. In the other prominent case dominated by various copper-oxalate complexes, $G$ changes on a different manner (Figure 1-Cu). When the oxalate and copper solutions are mixed at $t_{0}, G$ significantly drops (from $\sim 4500$ to $\sim 2500 \mu \mathrm{S}$ ) and reaches a value close to that of the the appropriate supersaturation for each reaction system in Figure 2a. The comparison of the exponents $\left(n_{S}\right)$ obtained by fitting eq 1 through each data series designates the species and reaction pathways playing major roles during precipitation (see the Supporting Information for the parameters of the fitted curves). However, to support such an $n_{S}$-based classification, the microstructure of the precipitate particles is investigated by scanning electron microscopy (see the Supporting Information for experimental details); relevant images are illustrated in Figure 3. Although the particle shape and size are consistent over the samples, no conclusion can be drawn by comparing the size of the crystals because different supersaturations, i.e., different reactant concentrations, are used for the distinct chemical systems in order to maintain comparable $t_{\text {ind }}$ values in each case. Nevertheless, Figure 3 shows an increasing geometrical complexity of the structures in conjunction with $n_{S}$. If $n_{S}$ is small, separated thin rods and small cubes compose the samples (Co, $\mathrm{Ni}, \mathrm{Zn}$, and $\mathrm{Mg}$ ). With an increasing exponent, the shape becomes more complex, as thick sheets (Ca) and dimpled bipyramids ( $\mathrm{Sr}$ ) are found. Importantly, these particles are still separated, which is a fingerprint of homogeneous nucleation. The most complex microfeatures are 

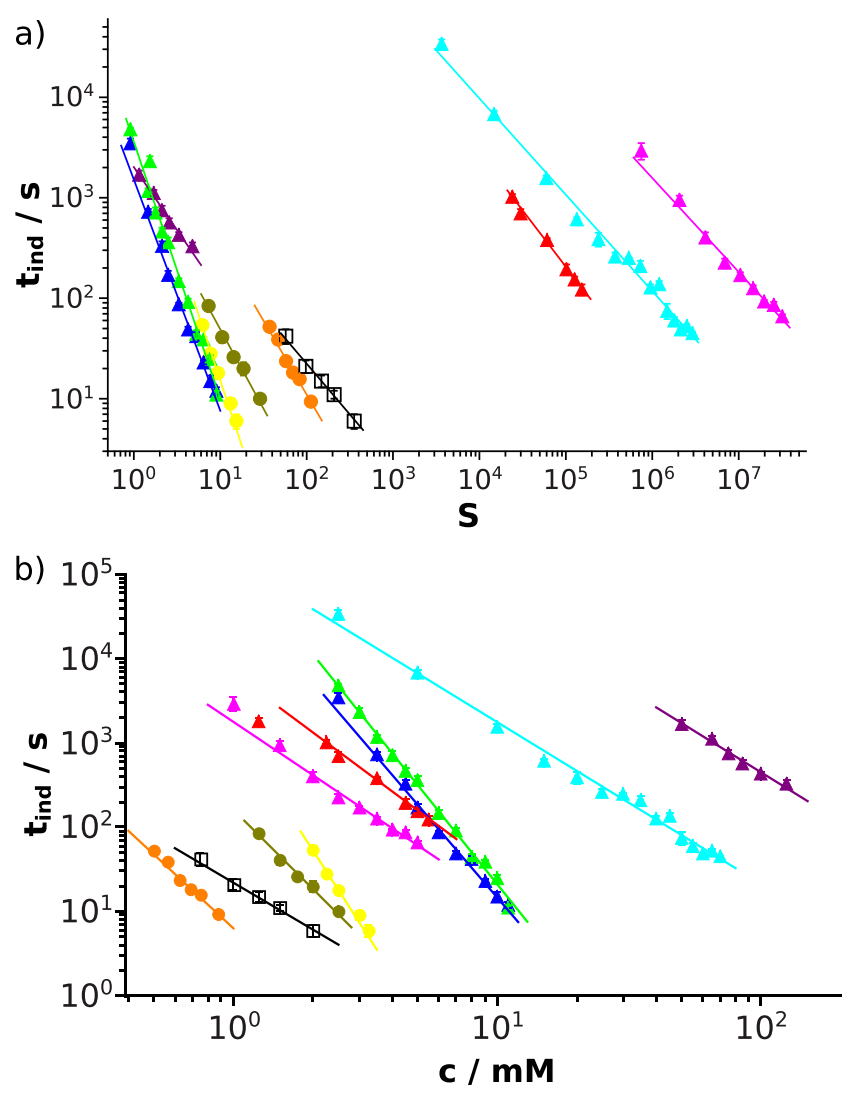

Figure 2. Induction period, $t_{\text {ind }}$, measured with a UV-vis spectrophotometer as a function of the supersaturation (a) and analytical concentration of the reactants (b) for various chemical systems: $\mathrm{MgCl}_{2}$ (purple $\boldsymbol{\Delta}$ ), $\mathrm{CaCl}_{2}$ (orange $\mathbf{\bullet}$ ), $\mathrm{SrCl}_{2}$ (olive $\mathbf{\bullet}$ ), $\mathrm{BaCl}_{2}$ (yellow 0 ), $\mathrm{CoCl}_{2}$ (magenta $\boldsymbol{\Delta}$ ), $\mathrm{NiCl}_{2}$ (light blue $\boldsymbol{\Delta}$ ), $\mathrm{CuCl}_{2}$ (dark blue $\mathbf{\Delta}$ ), $\mathrm{CuSO}_{4}$ (green $\mathbf{\Delta}$ ), $\mathrm{ZnCl}_{2}$ (red $\mathbf{\Delta}$ ), or $\mathrm{CdSO}_{4}$ (black $\square$ ) reacts with $\mathrm{Na}_{2} \mathrm{C}_{2} \mathrm{O}_{4}$ present in an equivalent concentration. Solid lines represent the fitted kinetic power laws; the parameters are tabulated in the Supporting Information.

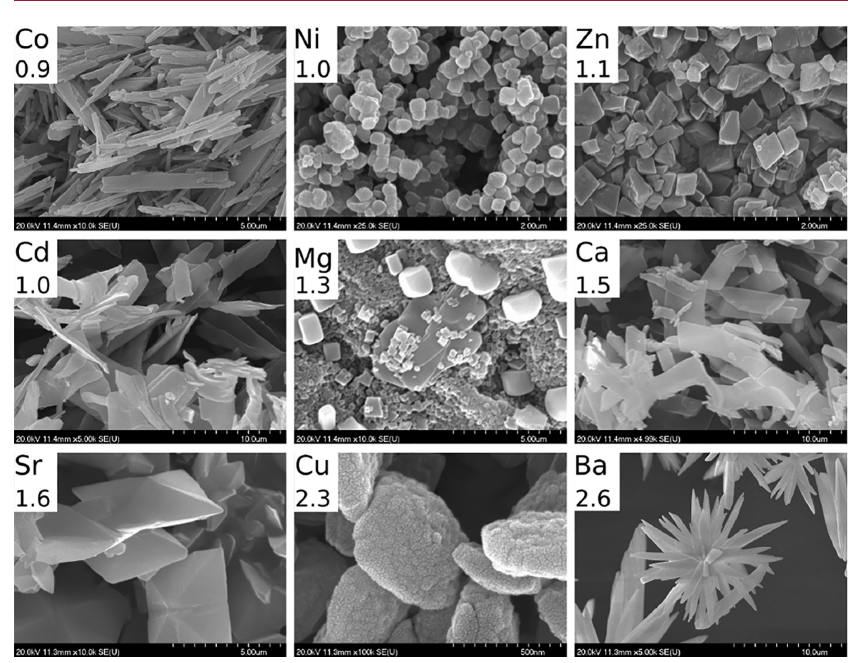

Figure 3. SEM micrographs of the precipitate microstructures arranged in ascending exponent, $n_{S}$, order.

present in the $\mathrm{Cu}$ and $\mathrm{Ba}$ systems, characterized by the largest exponents. The $\mathrm{Cu}$ sample is composed of hollow, pillowshaped aggregates of tiny crystals, while the Ba sample depicts a starlike structure built up by separate needles. These structures may refer to nonclassical nucleation and growth processes. Cd slightly stands out of the order because the thin sheets composing the sample would forecast an exponent larger than 1 .

The investigated chemical systems can be divided into two groups according to Figure $2 \mathrm{a}$. The reactions of $\mathrm{Co}, \mathrm{Ni}$, and $\mathrm{Zn}$ are separated from the other systems by means of the supersaturation required to reach a given induction period (e.g., $\left.10^{2} \mathrm{~s}\right)$; therefore, these reactions are now considered slow. The exponent $n_{S}$ is 1 within experimental error $( \pm 0.05)$ in each case. When these results are compiled, the conductance measurements proving the importance of complex formation and the equilibrium calculations highlighting the dominating role of the monooxalate complex, we can assume that precipitation mostly takes place via the neutral monooxalate complex-precipitate transformation as

$$
\mathrm{MC}_{2} \mathrm{O}_{4}(\mathrm{aq}) \rightleftharpoons \mathrm{MC}_{2} \mathrm{O}_{4}(\mathrm{~s})
$$

The very similar microfeatures of the separated crystals in a given chemical system let us assume homogeneous nucleation for each case. In the same way, the measured conductance drop emphasizes the role of native ions as precipitate precursors in the case of $\mathrm{Ca}$ and $\mathrm{Sr}$, which is further supported by the equilibrium calculations. An exponent remarkably different from the previous one is found, $n_{S}=1.5 \pm 0.1$ for both systems, which can be taken as the other limiting case mostly following an ionic pathway as

$$
\mathrm{M}^{2+}+\mathrm{C}_{2} \mathrm{O}_{4}^{2-} \rightleftharpoons \mathrm{MC}_{2} \mathrm{O}_{4}(\mathrm{~s})
$$

The reactions of $\mathrm{Ca}$ and $\mathrm{Sr}$ are considered fast, since a significantly lower $S$ is needed to provide $t_{\text {ind }} \approx 10^{2} \mathrm{~s}$ in comparison to the previous reactions and are taking place via homogeneous nucleation (see Figure 3).

The kinetic exponent of the remaining chemical systems and the expected reaction pathways are deduced from the two limiting cases. The reactions of $\mathrm{Mg}$ and $\mathrm{Cu}$ are surprisingly fast in comparison to the monooxalate precursor case, which emphasizes the importance of an ionic dioxalate pathway according to the equilibrium speciation. The negatively charged dioxalate complex may also react with the metal ion leading to the precipitation reaction

$$
\mathrm{M}^{2+}+\left[\mathrm{M}\left(\mathrm{C}_{2} \mathrm{O}_{4}\right)_{2}\right]^{2-} \rightleftharpoons 2 \mathrm{MC}_{2} \mathrm{O}_{4}(\mathrm{~s})
$$

in parallel to the pathway represented by eq 10 . This speculation is supported by $n_{S}=1.25 \pm 0.09$ and $2.30 \pm$ 0.11 determined for the $\mathrm{Mg}$ and $\mathrm{Cu}$ systems, respectively. Although the $\mathrm{Mg}$ case is characterized by a significantly lower exponent than the $\mathrm{Cu}$ case, it clearly differs both from the mostly monooxalate $\left(n_{S}=1\right)$ and from the mostly ionic $\left(n_{S}=\right.$ 1.5 ) pathways. Since $n_{S}=1.25$ is the arithmetic mean of those two limiting cases, the two parallel pathways are expected to be equally important. However, the pathways are separated because classical homogeneous nucleation is forecast by the microstructure (see Figure 3). The highest kinetic exponent is revealed for the $\mathrm{Cu}$ and $\mathrm{Ba}$ systems. Since the concentration of the dioxalate complex is the highest for $\mathrm{Cu}$ among the investigated systems (see the Supporting Information for percentage distributions), it is indeed expected to play a distinguishable role in the reaction. The exponent close to the sum of neutral monooxalate and ionic dioxalate pathways suggests that both reactions are important but in a different way from that seen in the case of $\mathrm{Mg}$. The pillow-shaped 
particle aggregates might be the result of nonclassical nucleation as a thermodynamic characteristic of the chemical system. This way, the product of one pathway acts as an activator of another pathway, which manifests in an exponent as a sum of the two: i.e., the reactions are coupled. Similarly to the $\mathrm{Cu}$ system, $\mathrm{Ba}$ also exhibits a high kinetic order $\left(n_{S}=2.60\right.$ \pm 0.18 ). According to the conductance measurements and equilibrium calculations, both pathways represented by eqs 10 and 11 might be important at the same time. A precipitation reaction proceeding on coupled pathways is further supported by the fact that the Ba system is slightly faster than the purely ionic $\mathrm{Ca}$ and $\mathrm{Sr}$ systems and is similarly as fast as the $\mathrm{Cu}$ system. However, the major difference is that simple ions (i.e., $\mathrm{Ba}^{2+}$ and $\mathrm{C}_{2} \mathrm{O}_{4}^{2-}$ ) play a role in the ionic pathway in the case of $\mathrm{Ba}$, while a complex ion $\left(\left[\mathrm{Cu}\left(\mathrm{C}_{2} \mathrm{O}_{4}\right)_{2}\right]^{2-}\right)$ is involved in the $\mathrm{Cu}$ system in addition to the monooxalate complexes.

Finally, the Cd system slightly deviates from the previously introduced order. Although both the equilibrium speciation and the conductance measurements prove that a considerable amount of monooxalate complex is produced, a significant amount of $\mathrm{M}^{2+}$ is also expected to be present and play a role. The determined kinetic exponent is 1 within experimental error, which indicates a reaction pathway according to eq 10 . Even though the precipitation is slower than those strongly incorporating the ionic step (e.g., $\mathrm{Cu}$ and $\mathrm{Ba}$ ), it is remarkably fast in comparison to the purely monooxalate systems $(\mathrm{Co}, \mathrm{Ni}$, and $\mathrm{Zn}$ ). This may suggest that the ionic pathway is also available to some small extent but it is not captured by the kinetic exponent, unlike the case for $\mathrm{Mg}$.

The adequate investigation of precipitation kinetics is based on supersaturation. ${ }^{20,21}$ However, the precipitate precursors are probably not the reactants directly brought into contact because of the various complex formation taking place in solution. As shown previously, confidently assigning the precursors is laborious and the results strongly rely on the quality of the chemical constants $\left(\beta_{1}, \beta_{2}, K_{\mathrm{sp}}\right.$, etc.) available in the literature. In addition, raw experimental data are usually obtained as $t_{\text {ind }}-c$ pairs, where $c$ is the analytical concentration of the given compound. Therefore, $t_{\text {ind }}=a_{c} c^{-n_{c}}$ function as a possible analogue of eq 1 is also fitted through the measured data points to shed light on the limitations of applying the analytical concentration instead of supersaturation. The fitted curves are illustrated in Figure $2 b$, and their parameters are tabulated in the Supporting Information for comparison. $n_{c}=$ $2 n_{S}$ is found in most cases with the exception of $\mathrm{Mg}$ and $\mathrm{Cu}$. Similarly to the trends revealed for $n_{S}$, the $\mathrm{Co}, \mathrm{Ni}$, and $\mathrm{Zn}$ systems which are characterized by the monooxalate pathway $\left(n_{S}=1\right)$ exhibit the same exponent within experimental error, for which $n_{c}=2 n_{S} \approx 2$ is determined. In the same way, $n_{c}=2 n_{S}$ $\approx 3$ describes the ionic pathway systems $\left(n_{S}=1.5\right.$ for Ca and $\mathrm{Sr})$. In the $\mathrm{Ba}$ case where parallel and coupled reactions are expected, since $n_{S}=2.5$ as a result of the pathways involving native ions and monooxalate complex, $n_{c}=2 n_{S}=2(1+1.5) \approx$ 5. As mentioned previously, Cd slightly deviates from the trend established on the basis of $n_{S}$, which remains for $n_{c}$ as well: i.e., the reaction is relatively fast in comparison to that forecast by the exponent. Nevertheless, $n_{c}=2 n_{S} \approx 2$ is still valid. In the remaining cases $(\mathrm{Mg}$ and $\mathrm{Cu}$ ) where parallel pathways are available via monooxalate and dioxalate complexes, $n_{c}<2 n_{S}$ is obtained. These findings show that, although more complex systems involving numerous complexes $(\mathrm{Mg}$ and $\mathrm{Cu})$ require the precise determination of supersaturation and thus the assignment of precipitate precursors, fitting as a function of the analytical concentration can provide valuable information about the time scale of the reaction and can indicate reaction pathways in simple cases. One may also notice that the plots shown in Figure 2a are meaningful for mechanism-scouting studies because the different systems fall into groups according to whether or not they incorporate ionic steps. On the other hand, the plots depicted in Figure $2 \mathrm{~b}$ have practical relevance in designing reaction mixtures in order to achieve a given range of induction periods. In addition, although Figure 3 depicts an $n_{S}$-based order, $n_{c}$ would provide the same trend, which further highlights the applicability of $t_{\text {ind }}-c$ curves.

The $t_{\text {ind }}-S$ curves obtained by taking various complex formations into account are tested by plotting theoretically derived kinetic equations to the $\mathrm{Ni}$ data set as proposed in the literature. ${ }^{20}$ The equations and the kinetic curves are presented in the Supporting Information for comparison. Although the formula deduced from the classical nucleation theory describes the data set, the fitted parameters have no physical meaning (negative values). The formula corresponding to polynuclear crystal growth mechanism, in the case when nucleation time $\left(t_{n}\right)$ is comparable to the time of a critical nucleus to reach a detectable size $\left(t_{g}\right)$, can only be fitted with meaningless parameters as well. Assuming a $t_{n} \ll t_{g}$ relation, no satisfactory fit is achieved with any proposed equation. In the opposite case, however, when $t_{n} \gg t_{g}$ is expected, the corresponding kinetic curve provides realistic surface energy for the nucleus $\left(\gamma_{s} \approx 0.15 \mathrm{~J} \mathrm{~m}^{-2}\right)$. This indicates a considerably long nucleation time in conjunction with the results of Figure 2a, proving that the $\mathrm{Ni}$ system belongs to the slower reactions. However, the fitted kinetic equation does not explain why the reaction rates are so different while the chemical systems are similar. In contrast, our method comparing $n_{S}$ values obtained by fitting eq 1 together with the results of equilibrium calculations is capable of revealing the differences in reaction mechanisms.

To exploit the potential of the complex precipitate shapes, fluoropolymer-based composite thin films containing $\mathrm{BaC}_{2} \mathrm{O}_{4}$ have been synthesized, where the precipitate particles play the role of polymer filler material (see the Supporting Information). The $\mathrm{BaC}_{2} \mathrm{O}_{4}$ particles with about $10-20 \mu \mathrm{m}$ characteristic size consist of radially standing needlelike crystals. Due to this special morphology, the particles exhibit a very rough surface structure and thus appear to be an ideal candidate for the design of water-repellent lotuslike coatings. To obtain superhydrophobic surfaces, a cooperative effect of low surface free energy and rough surface structures is necessary. ${ }^{26}$ The low surface energy of the synthesized composite layers is ensured by the fluoropolymer, while the adequate surface roughness is achieved by the $\mathrm{BaC}_{2} \mathrm{O}_{4}$ loading. Figure 4 shows SEM images of the composite layers with increasing $\mathrm{BaC}_{2} \mathrm{O}_{4}$ content. While the fluoropolymer layer without any precipitate content displays a relatively smooth surface (Figure $4 a$ ), the increasing oxalate content results in a significant increase in surface roughness (Figure $4 \mathrm{~b}, \mathrm{c}$ ). For 100 wt $\%$ precipitate content (no fluoropolymer), well-separated particles cover the substrate surface after spray coating (Figure $4 d)$. The effect of a varying surface roughness on the wetting properties of the synthesized layers is investigated by the contact angle $(\Theta$; see the Supporting Information) measurements (Figure $4 \mathrm{e}$ ). Without any oxalate filler, the initial hydrophobic fluoropolymer layer has a relatively smooth surface and $\Theta=104.3 \pm 4.8^{\circ}$, which is characteristic of lowenergy flat surfaces. ${ }^{27} \Theta$ increases significantly with an elevated 

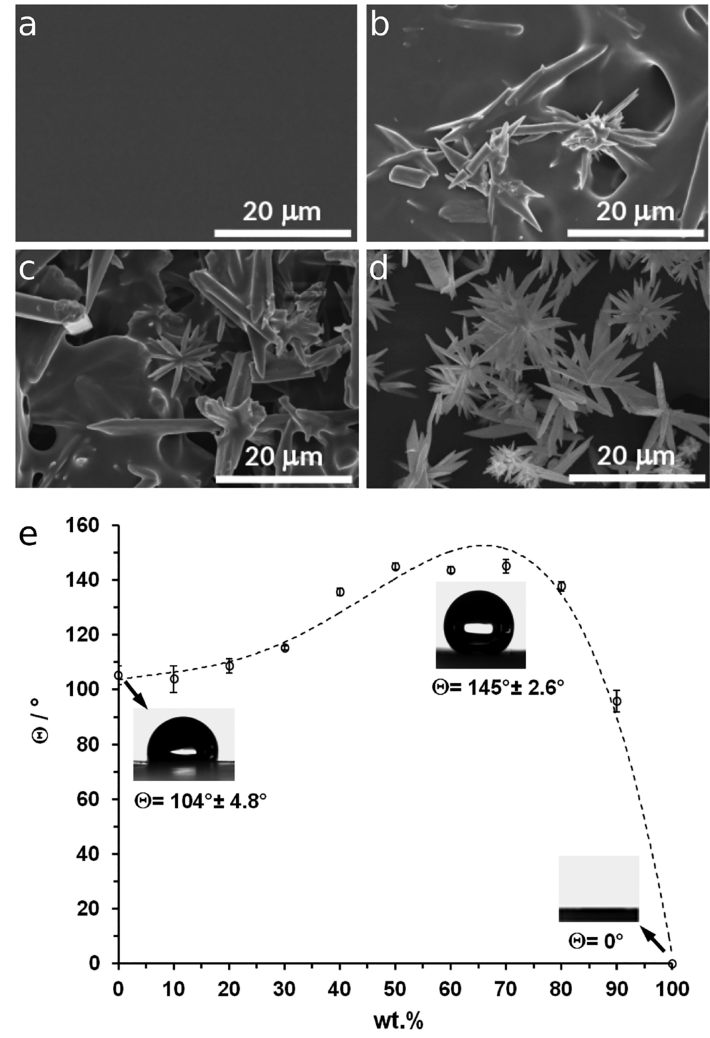

Figure 4. SEM micrographs of the fluoropolymer-based composite layers roughened by the addition of 0 (a), 20 (b), 50 (c), and 100 (d) wt $\%$ of $\mathrm{BaC}_{2} \mathrm{O}_{4}$ filler material. (e) Evolution of the apparent water contact angle $(\Theta)$ as a function of $\mathrm{BaC}_{2} \mathrm{O}_{4}$ filler material loading; snapshots of the static droplets also presented. A polynomial is fitted through the data points to better illustrate the trend.

precipitate loading and reaches its maximum $\left(145.0 \pm 2.6^{\circ}\right)$ at $70 \mathrm{wt} \%$ filler content. Such a value is only slightly below the superhydrophobic level $\left(\Theta>150^{\circ}\right)$. $\Theta$ sharply drops or equivalently the wetting increases by further increases in the filler content due to the hydrophilic character of the $\mathrm{BaC}_{2} \mathrm{O}_{4}$ particles.

\section{CONCLUSION}

It is shown that the exponent $n_{S}$ of the phenomenological power law traditionally used to describe the kinetics of precipitation reactions can provide information about the reaction mechanism and thus has chemical meaning. With the help of increasing $n_{S}$, the reaction mechanism of the investigated systems can be classified as $\mathrm{Co}, \mathrm{Ni}, \mathrm{Zn}$ (monooxalate pathway) < Mg (parallel pathways) $\mathrm{Ca}, \mathrm{Sr}$ (ionic pathway) $<\mathrm{Cu}, \mathrm{Ba}$ (coupled pathways). In addition, since determining supersaturation and assigning precipitate precursors are complicated in many chemical systems, we investigated to what extent the analytical concentration can be used instead of supersaturation. It is found that the same conclusions (reaction pathways and microstructure) can be drawn either way if the chemical system is not too combined. These findings will help to maintain a successful time scale matching between precipitation and coupled transport processes in order to facilitate tailored synthesis methods, leading to improved product properties and hierarchical precipitate structures. In addition to the possible reaction pathways, the kinetic exponent reflects the complexity of particle shapes as well; the higher the exponent, the more structured the particle.

\section{ASSOCIATED CONTENT}

\section{SI Supporting Information}

The Supporting Information is available free of charge at https://pubs.acs.org/doi/10.1021/acs.cgd.0c01061.

Experimental details of the turbidity and conductance measurements, experimental details of crystalline phase analysis and microstructure characterization, calculation of equilibrium speciation, supersaturation calculation with the fitted parameters of the kinetic curves, test of theoretically derived kinetic equations, and experimental details of surface wetting measurements (PDF)

\section{AUTHOR INFORMATION}

\section{Corresponding Author}

Gábor Schuszter - Department of Physical Chemistry and Materials Science, University of Szeged, Szeged H-6720, Hungary; ○orcid.org/0000-0002-9170-9933; Email: schuszti@chem.u-szeged.hu

\section{Authors}

Nirmali Prabha Das - Department of Physical Chemistry and Materials Science, University of Szeged, Szeged H-6720, Hungary

Réka Zahorán - Department of Physical Chemistry and Materials Science, University of Szeged, Szeged H-6720, Hungary

László Janovák - Department of Physical Chemistry and Materials Science, University of Szeged, Szeged H-6720, Hungary; orcid.org/0000-0002-2066-319X

Ágota Deák - Department of Physical Chemistry and Materials Science, University of Szeged, Szeged H-6720, Hungary

Ágota Tóth - Department of Physical Chemistry and Materials Science, University of Szeged, Szeged H-6720, Hungary; 구 orcid.org/0000-0001-8254-6354

Dezsö Horváth - Department of Physical Chemistry and Materials Science, University of Szeged, Szeged H-6720, Hungary; 10 orcid.org/0000-0003-3852-6879

Complete contact information is available at: https://pubs.acs.org/10.1021/acs.cgd.0c01061

\section{Notes}

The authors declare no competing financial interest.

\section{ACKNOWLEDGMENTS}

This work was supported by the National Research, Development and Innovation Office (K119795 and PD121010). L.J, and Á.D give special thank to the GINOP-2.3.2-15-201600013 and UNKP-19-4 New National Excellence Program of the Ministry For Innovation of Technology projects and to the János Bolyai Research Scholarship of the Hungarian Academy of Sciences. A 20391-3/2018/FEKUSTRAT grant of the Hungarian Ministry of Human Capacities is also acknowledged.

\section{REFERENCES}

(1) Aladjem, F.; Jaross, R. W.; Paldino, R. L.; Lackner, J. A. The antigen-antibody reaction. III. Theoretical Considerations Concerning the Formation, Location, and Curvature of the Antigen-Antibody PrecipitationZone in Agar Diffusion Plates, and a Method for the 
Determination of Diffusion Coefficients of Antigens andAntibodies. J. Immunol. 1959, 83, 221-231.

(2) Fan, C.; Li, J.; Xu, G.; He, H.; Ye, X.; Chen, Y.; Sheng, X.; Fu, J.; $\mathrm{He}$, D. FacileFabrication of Nano-hydroxyapatite/silk Fibroin Composite via a Simplified Coprecipitation Route. J. Mater. Sci. 2010, 45, 5814-5819.

(3) Hughes, E.; Williams, R.; Jenkins, M.; Grover, L. Formulation of a Covalently BondedHydroxyapatite and Poly(Ether Ether Ketone) Composite. J. Tissue Eng. 2018, 9, 1-13.

(4) Mascolo, M. C.; Pei, Y.; Ring, T. A. Room Temperature CoPrecipitation Synthesis of MagnetiteNanoparticles in a Large $\mathrm{pH}$ Window with Different Bases. Materials 2013, 6, 5549-5567.

(5) Khan, A. I.; O'hare, D. Intercalation Chemistry of Layered Double Hydroxides: RecentDevelopments and Applications. J. Mater. Chem. 2002, 12, 3191-3198.

(6) Handbook of Industrial Crystallization; Myerson, A., Erdemir, D., Lee, A., Eds.; Cambridge University Press: Cambridge, 2019; Chapter 8. DOI: $10.1017 / 9781139026949$.

(7) Zakhia Douaihy, R.; Al-Ghoul, M.; Hmadeh, M. Liesegang Banding for Controlled Size and Growth ofZeolitic-Imidazolate Frameworks. Small 2019, 15, 1901605.

(8) Bohner, B.; Schuszter, G.; Berkesi, O.; Horváth, D.; Tóth, Á. Self-organization ofCalcium Oxalate by Flow-driven Precipitation. Chem. Commun. 2014, 50, 4289.

(9) Ziemecka, I.; Gokalp, S.; Stroobants, S.; Brau, F.; Maes, D.; De Wit, A. Polymorph Selectionof ROY by Flow-driven Crystallization. Crystals 2019, 9, 351.

(10) Ding, Y.; Batista, B.; Steinbock, O.; Cartwright, J. H. E.; Cardoso, S. S. S. Wavy Membranes andthe Growth Rate of a Planar Chemical Garden: Enhanced Diffusion and Bioenergetics. Proc. Natl. Acad. Sci. U. S. A. 2016, 113, 9182-9182.

(11) Cardoso, S. S. S.; Andres, J. T. H. Geochemistry of Silicate-rich Rocks Can Curtail Spreading ofCarbon Dioxide in Subsurface Aquifers. Nat. Commun. 2014, 5, 5743.

(12) Moosavi, R.; Kumar, A.; De Wit, A.; Schröter, M. Influence of Mineralization and InjectionFlow Rate on Flow Patterns in Threedimensional Porous Media. Phys. Chem. Chem. Phys. 2019, 21, 14605.

(13) Vekilov, P. G. Crystallization Tracked Atom by Atom. Nature 2019, 570, 450-451.

(14) Vekilov, P. G. Nucleation. Cryst. Growth Des. 2010, 10, 50075019.

(15) Xyla, A. G.; Giannimaras, E. K.; Koutsoukos, P. G. The Precipitation of Calcium Carbonate inAqueous Solutions. Colloids Surf. 1991, 53, 241-255.

(16) Jiang, S.; ter Horst, J. H. Crystal Nucleation Rates from Probability Distributions ofInduction Times. Cryst. Growth Des. 2011, 11, 256-261.

(17) Xiao, Y.; Wang, J.; Huang, X.; Shi, H.; Zhou, Y.; Zong, S.; Hao, H.; Bao, Y.; Yin, Q. Determination Methods for Crystal Nucleation Kinetics in Solutions. Cryst. Growth Des. 2018, 18, 540-551.

(18) Das, N. P.; Müller, B.; Tóth, Á.; Horváth, D.; Schuszter, G. Macroscale precipitationkinetics: Towards complex precipitate structure design. Phys. Chem. Chem. Phys. 2018, 20, 19768.

(19) Zahorán, R.; Kukovecz, Á.; Tóth, Á.; Horváth, D.; Schuszter, G. High-speed Trackingof Fast Chemical Precipitations. Phys. Chem. Chem. Phys. 2019, 21, 11345-11350.

(20) Söhnel, O.; Mullin, J. W. Interpretation of Crystallization Induction Periods. J. Colloid Interface Sci. 1988, 123, 43-50.

(21) Kashchiev, D.; van Rosmalen, G. M. Review: Nucleation in Solutions Revisited. Cryst. Res. Technol. 2003, 38, 555-574.

(22) Mullin, J. W.Crystallization, 4th ed.; Butterworth-Heinemann: Oxford, 2001.

(23) Kotrlý, S.; Šicha, L. Handbook of Chemical Equilibria in Analytical Chemistry; Ellis Horwood: 1985.

(24) Altomare, A.; Corriero, N.; Cuocci, C.; Falcicchio, A.; Moliterni, A.; Rizzi, R. QUALX2.0: Aqualitative phase analysis software using the freely available database POW-COD. J. Appl. Crystallogr. 2015, 48, 598-603.
(25) Ni, L.; Wang, L.; Shao, B.; Wang, Y.; Zhang, W.; Jiang, Y. Synthesis of flower-like zincoxalate microspheres in ether-water bilayer refluxing systems and their conversion to zinc oxide microspheres. J. Mater. Sci. Technol. 2011, 27, 563-569.

(26) Mérai, L.; Deák, Á.; Sebők, D.; Csapó, E.; Kolumbán, T. S.; Hopp, B.; Dékány, I.; Janovák, L. Photoreactive composite coating with composition dependent wetting properties. eXPRESS Polym. Lett. 2018, 12, 1061-1071.

(27) Miwa, M.; Nakajima, A.; Fujishima, A.; Hashimoto, K.; Watanabe, T. Effects of the surfaceroughness on sliding angles of water droplets on superhydrophobic surfaces. Langmuir 2000, 16, 5754-5760. 